\title{
Intracranial infections: lessons learned from 52 surgically treated cases
}

\author{
Cahit Kural, MD, ${ }^{1}$ Sahin Kırmızıgoz, MD, ${ }^{1}$ Mehmet Can Ezgu, MD, ${ }^{1}$ Orhan Bedir, MD, ${ }^{2}$ \\ Murat Kutlay, MD, ${ }^{1}$ and Yusuf Izci, MD ${ }^{1}$ \\ Departments of ${ }^{1}$ Neurosurgery and ${ }^{2}$ Microbiology, University of Health Sciences, Gulhane School of Medicine, Ankara, Turkey
}

OBJECTIVE Intracranial infections are serious and life-threatening health problems. They may present as subdural empyemas or intracerebral abscesses. Surgical drainage and subsequent antibiotic treatment is the main technique for a satisfactory clinical outcome. The aims of this study were to present a 10 -year intracranial infection series and discuss the surgical characteristics in the light of literature.

METHODS Fifty-two patients with intracranial infection underwent surgical treatment between 2008 and 2018. Eleven patients were female and 41 patients were male. The mean age was 40.46 years (range 10-75 years). Eighteen patients had intracerebral abscesses, and 34 had subdural empyemas. All patients underwent surgical treatment as well as an antibiotic regimen.

RESULTS No etiological agent was isolated in 29 (56\%) cases. Bacterial agents were detected in 20 cases, while fungi were observed in 3 cases. Staphylococci species were the most common agents and were isolated in $8(15 \%)$ cases. Endoscopic aspiration was performed in 3 cases, while surgical drainage and capsule resection via craniotomy was performed in 49 cases. An associated intracranial tumor was diagnosed in 2 patients with brain abscesses. Four (8\%) patients died despite surgical and medical treatments.

CONCLUSIONS Surgical treatment via craniotomy is an older method, but it is still the best to treat the intracranial infections not only for decompression of the brain but also to attain an accurate diagnosis. The abscess wall should always be histologically examined after surgery to rule out any intracranial tumor.

https://thejns.org/doi/abs/10.3171/2019.5.FOCUS19238

KEYWORDS infection; abscess; empyema; surgery

I NTRACRANIAL infections include a wide range of different medical conditions, each with unique clinical, radiological, and surgical characteristics. ${ }^{13,26}$ Many of these conditions progress rapidly and result in significant morbidity and mortality if appropriate diagnostic and therapeutic procedures are not initiated. ${ }^{13}$ Our study is focused on intracerebral (brain) abscesses and subdural empyemas, which are the most surgically treated intracranial infections.

A brain abscess is a focal pyogenic infection of the brain's parenchyma. It has a typical capsule formation and purulent material inside the capsule. Brain abscesses may be solitary lesions or present as multiple intracranial foci. ${ }^{11}$
The most frequent locations are the frontotemporal, frontoparietal, parietal, and occipital lobes and the cerebellum. ${ }^{4}$ Although brain abscesses are mostly seen in adults, they may also present in childhood. ${ }^{11}$ Major predisposing factors for the development of a brain abscess are a close infection focus, hematogenous spread from a distant focus, and previous trauma. ${ }^{625}$ The microbiological etiology of the abscess depends on many factors such as the site of primary infection, a patient's age and immunological condition, underlying diseases, and the geographic location of the patient. ${ }^{12}$

Subdural empyema is also a pyogenic infection but is located between the dura and arachnoid matres. It is an 


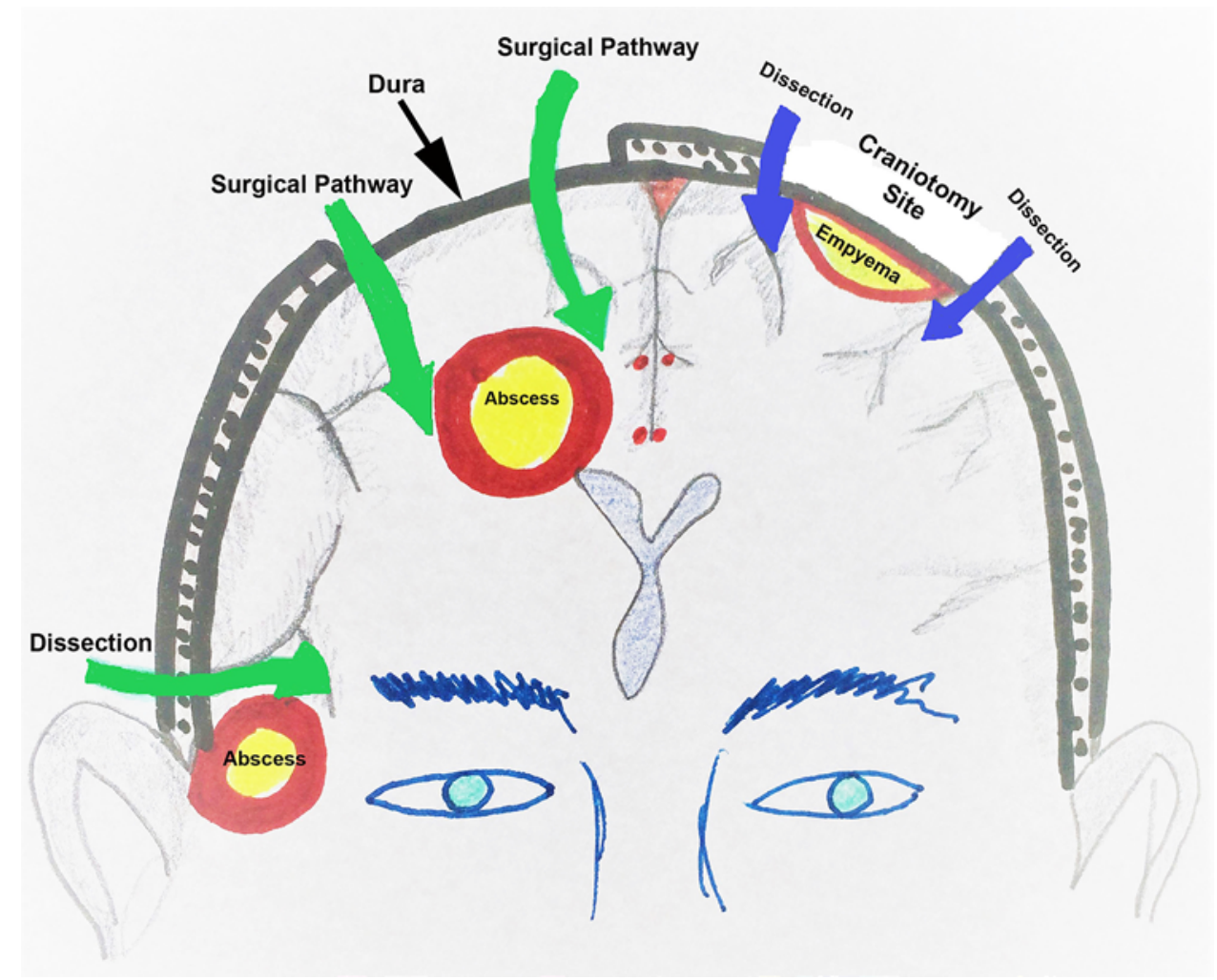

FIG. 1. Illustration showing the craniotomy and approach techniques to the intracerebral abscesses and subdural empyemas. The surgical pathways (green arrows) for intracerebral abscesses should always aim to dissect abscess walls from surrounding brain tissue. The dissection (blue arrows) of the inner capsule of subdural empyemas is always difficult.

uncommon condition, representing $15 \%-20 \%$ of confined intracranial diseases. ${ }^{14}$ The most common cause is a previous neurosurgical procedure, such as tumor or subdural hematoma surgery. Sinusitis and otogenic infections are the other etiological factors. Previous skull trauma and meningitis may also be responsible for subdural empyemas..$^{14,23}$

CT scanning and MRI are the two primary radiological modalities used for the detection of intracranial infections. ${ }^{13,25}$ While CT scanning is widely available and very useful for rapid diagnosis and assessment of hydrocephalus, mass lesions, hemorrhage, or brain edema, MRI is often required to detect more subtle findings. MRI is more sensitive, especially for evaluating CSF involvement, leptomeningitis, empyema, ventriculitis, vasculitis, and abscess. ${ }^{1,5,13,18}$ Metastatic brain tumors and radiation necrosis are the most common conditions in the differential diagnosis of intracranial infections. . $^{1,10,28}$

Current surgical treatment methods include craniotomy for resection or stereotactic aspiration of the abscess material. ${ }^{7,15}$ In most instances, drainage of the purulent material is enough to initiate healing of the intracranial infection. ${ }^{1}$ In addition, there is no significant difference between excision and drainage alone in terms of patient morbidity and mortality. However, excision is better than aspiration with regard to duration of antibiotic use, length of hospital stay, and overall cost of treatment. ${ }^{1}$ Endoscopic approaches are being used for deep-seated abscesses. ${ }^{24}$
Resection using craniotomy is usually the preferred technique for large, multiple abscesses causing mass effect or superficial lesions located in noneloquent brain regions. ${ }^{3,728}$ Stereotactic aspiration is reserved for deepseated abscesses or those located within or adjacent to eloquent regions. ${ }^{10,24,25}$

We conducted a retrospective review of the intracranial infections treated in our department over the past 10 years. We focused on patients with brain abscess or subdural empyema who underwent surgical intervention. Surgical techniques and postoperative outcomes in particular are presented and discussed.

\section{Methods}

This is a retrospective study from a neurosurgery department of a tertiary university hospital. Fifty-two patients with intracranial infection (brain abscess or subdural empyema) underwent surgical treatment between 2008 and 2018. Eleven patients were female, and 41 (79\%) patients were male. The mean age was 40.46 years (range 10-75 years). MRI was used for the diagnosis and followup of all patients. The surgical strategy was designed based on the radiological characteristics of each case. The important part of our surgical technique was removal of the abscess wall either partially or totally, if possible. Small abscesses were removed en bloc once the cleavage plane was identified, while large abscesses were drained before 

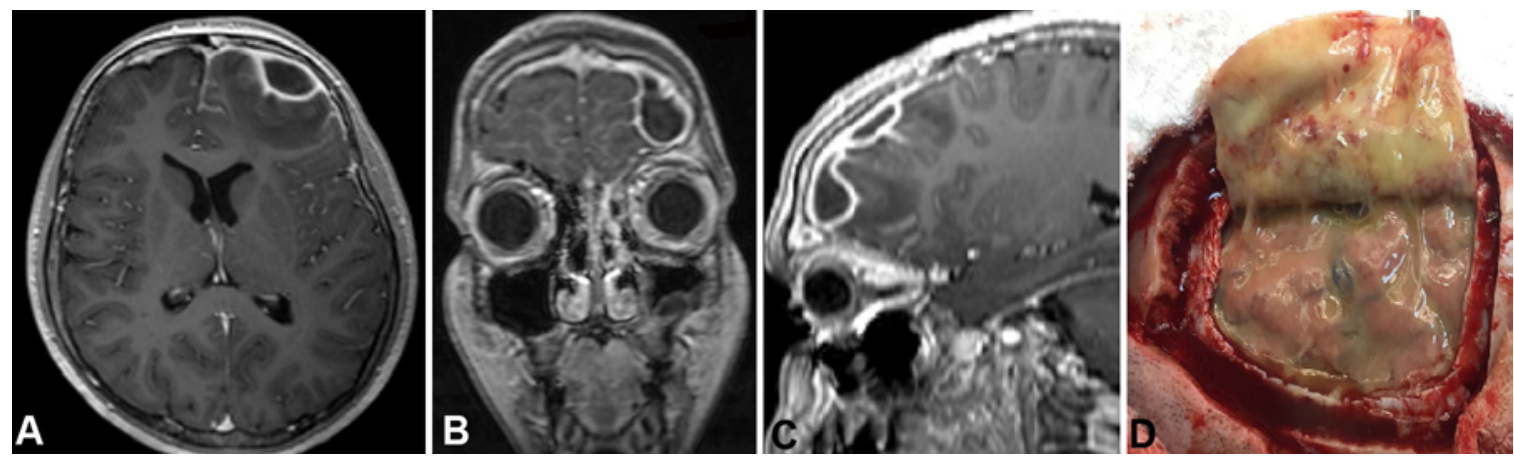

FIG. 2. Contrast-enhanced axial (A), coronal (B), and sagittal (C) T1-weighted MRI scans obtained in a patient with a left frontal subdural empyema due to previous cranial surgery. The empyema was removed using craniotomy, and green purulent material was drained. The inner capsule was thick and difficult to remove or perforate (D).

a secure plane was found to limit the risks of damage to the surrounding brain tissue. Craniotomy was preferred for subdural empyema and most of the brain abscesses. It was used to reach and expose the entire wall of the brain abscess. Some abscess walls were rigid and dense, while the others were thin and difficult to dissect from the cortex. Patients in early or late cerebritis stages had very thin abscess walls. In these cases, the surgical pathway was designed to extend the exposure of the abscess wall, with special attention to the surrounding vascular and neural structures (Fig. 1). Cerebral sulci close to the cerebral abscess were used for exposure of the abscesses. A neuronavigation system was used to provide easy access to deep abscesses and drainage of the infection material with less invasive methods. However, we never performed drainage alone, and the abscess wall was always partially or totally removed during the surgery. This was the critical part of the procedure for the prevention of abscess recurrence and for differentiation of brain abscesses from tumor, especially brain metastases. Drainage of the entire infection material was not possible in some cases due to the deep location of the abscess, significant brain edema, or proximity to important neurovascular structures. In these cases, even if the abscess material could not be completely drained, some samples of the abscess wall were obtained for histopathological examination. In subdural empyema cases, craniotomy allowed a wide exposure, adequate exploration, and better evacuation of the purulent material (Fig. 2). It was easy to reach and evacuate the infection material via craniotomy. However, dissection and removal of the thick and adherent inner membrane was quite difficult. In most cases, the inner membrane was partially removed because of the risk of cortical damage (Fig. 1). The color of the material was also recorded for the differentiation of the abscess etiology, as green abscess material (Fig. 2), yellowish material, and white material may be indicators of different microorganisms (Figs. 3-5). Grayish material was observed in cases of necrosis, which was secondary to previous radiotherapy or malignant brain tumors such as glioblastoma.

Endoscopic navigation-based abscess drainage was performed in selected patients with deep-seated abscess. The neuronavigation system was used for all intracranial abscess cases. Etiology, clinical presentations, Glasgow
Coma Scale score at admission, length of hospital stay, and culture results obtained were recorded for all patients.

This study was approved by the University of Health Sciences, Gulhane noninterventional research ethics committee.

\section{Results}

The characteristics of 52 patients are summarized in Table 1. Eighteen (35\%) patients had intracerebral abscesses, and 34 had subdural empyemas. Sinusitis, previous cra-
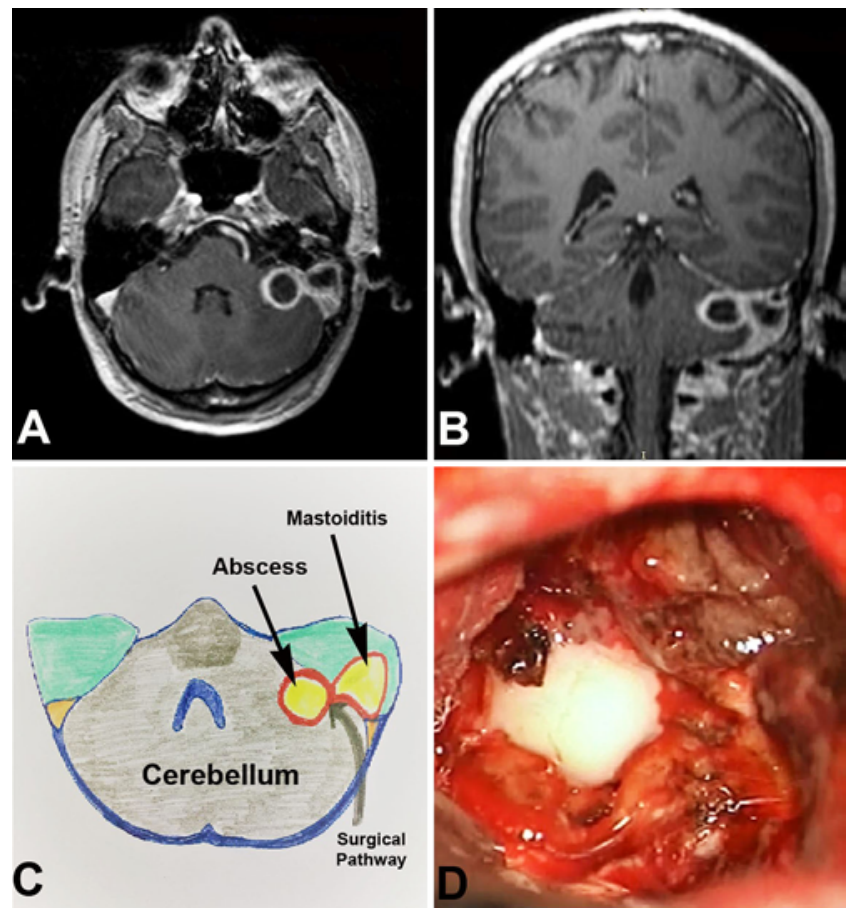

FIG. 3. Contrast-enhancing axial (A) and coronal (B) T1-weighted MRI scans obtained in a patient with a left cerebellar abscess and left temporal mastoiditis. The left retrosigmoid approach was used (C), and the yellow abscess material was drained (D). Then, the mastoid part of the temporal bone was drilled, and the infection material within the bone was removed. 

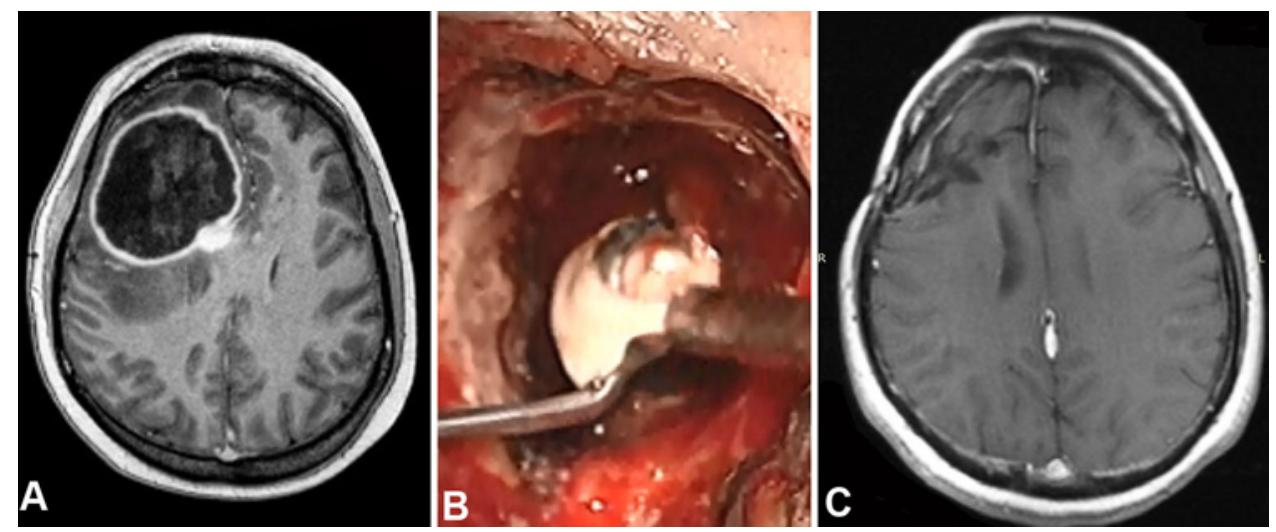

FIG. 4. Contrast-enhanced axial T1-weighted MRI scan showing a right frontal giant intracerebral abscess (A). The white infection material was removed using forceps (B). This case was an Aspergillus abscess. Axial postoperative MRI scan confirms complete removal of the abscess (C).

niotomy, otorhinogenic infection (Fig. 3), and trauma (Fig. 6) were the common causes of intracranial infection in our series. No microbiological agent was isolated in $29(56 \%)$ cases, while staphylococci species were isolated in 8 cases. Endoscopic navigation-based aspiration was performed in 3 cases (Fig. 7), and abscess removal via craniotomy was performed in 15 intracerebral abscess cases (Fig. 1). The mean length of hospital stay was 33.7 days (range 4-68 days) for patients with brain abscesses and 27.3 days (range 7-130 days) for patients with subdural empyemas. Three patients with abscesses died after surgery, and 1 patient died after subdural empyema drainage. Two of 3 abscess patients were immunocompromised; in addition, one had multiple Aspergillus abscesses and the other had a left thalamic abscess (Fig. 7). Both patients died within 1 week after surgery. The other 2 patients died of previously diagnosed malignant tumors within 1 year postoperatively. Aspergillus was detected in 2 brain abscess cases, and both of these patients had multiple abscesses (Fig. 4). Mucormycosis was isolated in 1 frontobasal brain abscess. Associated intracranial tumor was diagnosed in 2 patients with brain abscess. One was a chordoma (Fig. 5) and the other was an anaplastic astrocytoma.

\section{Discussion}

We presented 52 patients who underwent surgical treatment for intracranial infection. Eighteen $(35 \%)$ of the patients had intracerebral abscesses while the remaining patients had subdural empyemas secondary to previous surgeries, implanted materials, and otogenic infections. Most of the patients were male, and most patients were between the ages of 18 and 39 years. Two patients had an intracranial tumor associated with the abscess. No etiological agent could be identified in $29(56 \%)$ cases, while staphylococci species were the most common agents in our series. The mortality rate was $7.7 \%$.

Otogenic brain abscess and meningitis are the most widely recognized focal complications of otitis media. ${ }^{8}$ Curiously, an otogenic brain abscess identified with otitis media is usually found nearby the temporal bone, mostly within the temporal lobe and cerebellum (Fig. 3). This area is additionally demonstrative of the principal mechanism of infection that advances through the osseous borders of temporal bone by means of osteitis and bony erosion. In these cases, the abscess can be drained through the transmastoid approach, avoiding an extra craniotomy. ${ }^{27}$ In our series, otorhinogenic infection was detected as the prima-
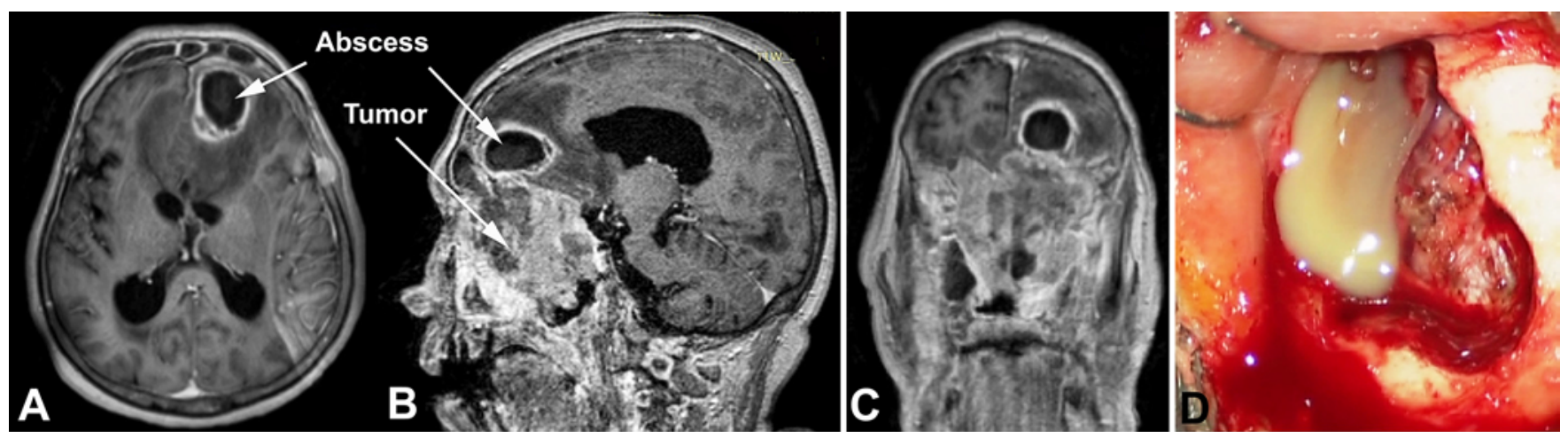

FIG. 5. Contrast-enhanced axial (A), sagittal (B), and coronal (C) T1-weighted MRI scans obtained in a patient with a left frontal intracerebral abscess and craniofacial chordoma. The yellow abscess material was first drained, and then the tumor was removed in the same session (D). 
TABLE 1. Clinical characteristics of 52 patients with intracranial infections

\begin{tabular}{|c|c|}
\hline Characteristic & No. of Patients (\%) \\
\hline \multicolumn{2}{|l|}{ Sex } \\
\hline Male & $41(79)$ \\
\hline Female & $11(21)$ \\
\hline \multicolumn{2}{|l|}{ Age, yrs } \\
\hline $10-17$ & $2(4)$ \\
\hline $18-39$ & $25(48)$ \\
\hline $40-59$ & $13(25)$ \\
\hline $60-75$ & $12(23)$ \\
\hline \multicolumn{2}{|l|}{ Presenting symptoms } \\
\hline Headache & $34(65)$ \\
\hline Nausea/vomiting & $16(31)$ \\
\hline Confusion, drowsiness & $5(10)$ \\
\hline Neurological deficits & $4(8)$ \\
\hline Seizures & $3(6)$ \\
\hline \multicolumn{2}{|l|}{ GCS score on admission } \\
\hline $15-14$ & $44(85)$ \\
\hline $13-8$ & $6(12)$ \\
\hline $7-3$ & $2(3)$ \\
\hline \multicolumn{2}{|l|}{ Predisposing factor } \\
\hline Sinusitis & $13(25)$ \\
\hline Previous craniotomy & $10(20)$ \\
\hline Otorhinogenic infection & $8(15)$ \\
\hline Trauma & $7(13)$ \\
\hline Immunocompromised & $5(10)$ \\
\hline None identified & $9(17)$ \\
\hline \multicolumn{2}{|l|}{ Infection type } \\
\hline Subdural empyema & $34(65)$ \\
\hline Single brain abscess & $14(27)$ \\
\hline Multiple brain abscesses & $4(8)$ \\
\hline \multicolumn{2}{|l|}{ Infection location } \\
\hline Frontal & $15(29)$ \\
\hline Temporal & $7(13)$ \\
\hline Parietal & $9(17)$ \\
\hline Occipital & 2 \\
\hline Cerebellar & 4 \\
\hline Frontoparietal & 4 \\
\hline Frontotemporal & 3 \\
\hline Thalamic & 1 \\
\hline Frontobasal & 1 \\
\hline Brainstem & 1 \\
\hline Sellar & 1 \\
\hline Multiple & 4 \\
\hline \multicolumn{2}{|l|}{ Culture } \\
\hline No organism identified & $29(56)$ \\
\hline Staphyloccocus aureus & $5(10)$ \\
\hline Staphyloccocus epidermidis & 3 \\
\hline Streptococcus pneumoniae & 2 \\
\hline Streptococcus sapprophyticus & 1 \\
\hline
\end{tabular}

CONTINUED IN NEXT COLUMN
" CONTINUED FROM PREVIOUS COLUMN

TABLE 1. Clinical characteristics of 52 patients with intracranial infections

\begin{tabular}{cc}
\hline Characteristic & No. of Patients (\%) \\
\hline Culture (continued) & \\
\hline Streptococcus constellatus & 1 \\
\hline Streptococcus anginosus & 1 \\
\hline Klebsiella pneumoniae & 2 \\
\hline Pseudomonas aeruginosa & 1 \\
\hline Enterococcus faecalis & 1 \\
\hline Parvimonas micra & 1 \\
\hline Aspergillus & 2 \\
\hline Mucormycosis & 1 \\
\hline Multiple organisms & $2(4)$ \\
\hline
\end{tabular}

GCS = Glasgow Coma Scale.

ry source in 8 patients. One of these 8 patients had a simultaneous cerebellar abscess and mastoiditis, and the patient underwent removal of abscess and drainage of the mastoiditis in the same session. We performed a suboccipital craniotomy and retrocerebellar approach to reach and remove the abscess. Then, the mastoid bone was drilled, and purulent material within the bone was drained (Fig. 3).

Differential diagnosis of brain abscesses is of paramount importance. Metastatic brain tumors and radiation necrosis are the most common conditions in the differential diagnosis. Dynamic susceptibility contrast-enhanced perfusion MRI, proton MR spectroscopy, and diffusionweighted imaging can be used preoperatively to differentiate abscesses from glioblastomas and/or metastases. ${ }^{21,29}$ A brain abscess may be associated with or located within the brain tumor. This is a very rare occurrence, and only a few cases of abscess associated with intracranial tumor have been reported in the literature. The mortality of this combination is quite high. The most reported tumors coexisting with abscesses are glioblastomas, but meningiomas, astrocytomas, and metastases have also been reported..$^{2,9,16,19,21}$ The exact pathophysiological basis of this coexistence is unknown, but immunodeficiency of the patients due to the immunosuppressive agents given for cancer treatment may contribute to the coexistence of abscess and metastasis. ${ }^{16}$ Another mechanism is that the tumor necrosis cavity may be a potential medium for an infection to develop. ${ }^{25}$ Moreover, if the tumor is close to the venous sinuses, hematogenous transmission of bacterial pathogens may be facilitated from the venous circulation to the tumor. ${ }^{6}$ Goto et al. ${ }^{16}$ reported a case of brain abscess within the cerebellar metastasis, and they emphasized the importance of considering coexisting diseases prior to surgery. They also showed the importance of abscess wall resection and examination for the accurate diagnosis. In our series, 2 patients had associated intracranial tumors. One had a chordoma and the other had a cystic anaplastic astrocytoma. The chordoma was closely associated with a frontal abscess, which were both removed during the same session (Fig. 5). 

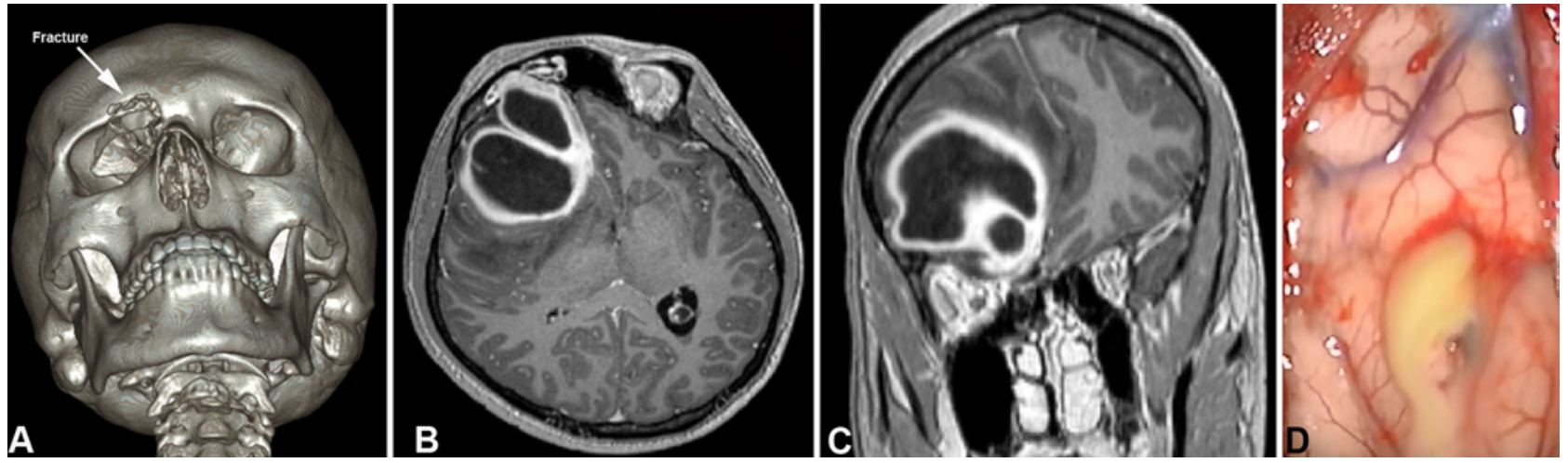

FIG. 6. A case of right frontal abscess due to a previous orbitofrontal fracture. Three-dimensional CT scan showing the right orbitofrontal fracture (A). Contrast-enhanced axial (B) and coronal (C) T1-weighted MRI scans showing a right frontal bi-lobulated intracerebral abscess. The yellow infection material was drained, and the capsule was removed via a right craniotomy (D).

Bacteroides, Peptostreptococcus, and Streptococcus are mostly isolated in brain abscesses secondary to contiguous dissemination. ${ }^{4,10,25}$ Peptostreptococcus and Streptococcus (especially viridans streptococci and microaerophilic streptococci) are mostly identified in patients with a brain abscess due to cardiac disease..$^{25}$ Staphylococcus and Streptococcus are found in patients who underwent previous surgical procedures. Staphylococcus, Streptococcus, Clostridium, and Enterobactericeae are seen in patients with open head trauma. ${ }^{4,22,25}$ Fungal infections, Toxoplasma, Staphylococcus species, Streptococcus species, and Pseudomonas are frequently observed in immunocompromised patients and after organ transplantation, chemotherapy, and steroid usage..$^{4,20,25}$ Streptococcus anginosus is a commensal microbe of the mucosae of the oral cavity, gastrointestinal tract, and urogenital tract and is rarely found in brain abscess materials or immunocompetent patients. ${ }^{12}$ We identified this etiological agent in an immunocompetent patient with a left frontal intracerebral abscess that was treated successfully with craniotomy and drainage. In our series, a microbial agent was not identified in most cases. Staphylococci species were the most common microbial agents that caused intracranial infection, followed by streptococci species. Aspergillus was isolated in $2 \mathrm{im}$ - munocompromised patients due to malignancies (Fig. 4). We inserted amphotericin-B in the abscess cavity during surgery following removal of the infection material. This technique was previously reported in the literature with satisfactory clinical results. ${ }^{20}$

Surgical options for the treatment of brain infections are well known and defined in the literature. ${ }^{3,7,10,15,17}$ Craniotomy is the gold standard for superficial, large, or multiloculated abscess or empyemas while stereotactic methods or endoscopic approaches are preferred for deep-seated or small infections, ${ }^{7,24}$ According to Moosa et al., ${ }^{24}$ endoport-assisted surgical resection of deep-seated cerebral abscesses is feasible, safe, and effective in appropriately selected patients. The endoport system provides adequate visualization of deep abscesses, which allows extensive excision and drainage while minimizing parenchymal damage and white matter retraction. We used this technique in 2 cases in combination with the neuronavigation system, and the abscess material was evacuated using the endoscopic technique (Fig. 7). Although endoport-assisted resection provides easy abscess drainage, it is not enough to perform abscess wall perforation for the prevention of recurrence. Craniotomy plus microsurgery is more effective and safer for abscess wall perforation and drainage.
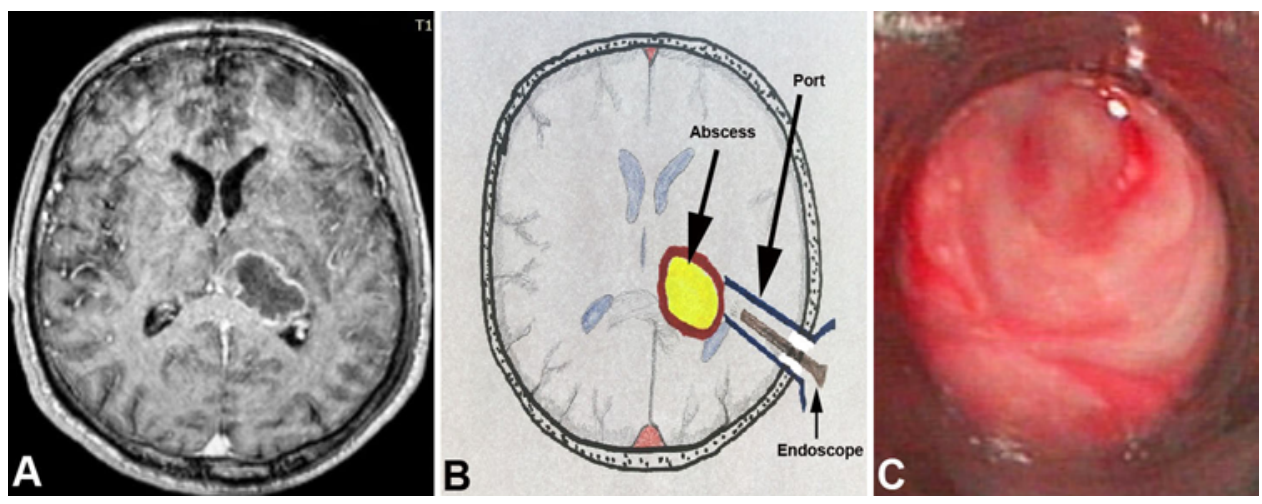

FIG. 7. Contrast-enhanced axial T1-weighted MRI scan showing a left thalamic abscess (A). The abscess was drained using the endoport system and endoscope (B and $\mathbf{C})$. 
This method also provides clear exposure of the abscess environment for washing with antimicrobial agents.

Adequate drainage of the infection material (pus and capsule) leads to clinical improvement as well as neurological and hemodynamic stability. ${ }^{25}$ The main aim of surgery is to drain the entire infection material and to decompress the brain in a single attempt, but this goal is seldom achieved with endoscopic or stereotactic methods. In the case of a dense or strong abscess wall, infection material can be left in situ, but abscess wall sampling is crucial for histopathological examination.

A subdural empyema can be misdiagnosed as a subdural hematoma or fluid collection. ${ }^{31}$ Contrast-enhanced radiological examinations are necessary for the differential diagnosis of subdural empyema (Fig. 2). In the case of a large amount of brain edema associated with fever and elevated white blood cells and sedimentation rate, radiological examinations should always be performed with contrast agents. Previous neurosurgical procedures, heart disease, and trauma should also be included in the patient's medical history for an accurate diagnosis. ${ }^{13,14,23}$ Moreover, subdural empyemas are a causal factor in the pathogenesis of cerebral venous thrombosis in adults. ${ }^{30}$ Therefore, these lesions should be treated urgently in order to avoid cortical vein thrombosis or cerebral infarction. In our series, 34 of 52 patients had subdural empyema and underwent surgical treatment via craniotomy. All operations were performed on an emergency basis, and only 1 patient died after surgery. We did not observe any case of cerebral infarction secondary to subdural empyema in our series.

This study has several limitations: first is its retrospective nature, second is the low number of cases, and third is the lack of a sufficient follow-up period for a better clinical outcome.

\section{Conclusions}

Surgical treatment of intracranial infections requires good surgical skills, experience, and anatomical knowledge. Each case should be managed individually according to the patient's clinical and radiological features. Complete drainage of the material should always be balanced with the anatomical location of the infection. Sampling and histological examination of the abscess wall is of paramount importance for an accurate diagnosis.

\section{References}

1. Alvis Miranda H, Castellar-Leones SM, Elzain MA, Moscote-Salazar LR: Brain abscess: current management. J Neurosci Rural Pract 4 (Suppl 1):S67-S81, 2013

2. Bansal S, Vasishta RK, Pathak A, Jindal VN, Khosla VK, Banerjee AK: Cerebral abscess with astrocytoma. Neurol India 49:91-93, 2001

3. Beller AJ, Sahar A, Praiss I: Brain abscess. Review of 89 cases over a period of 30 years. J Neurol Neurosurg Psychiatry 36:757-768, 1973

4. Brook I: Microbiology and treatment of brain abscess. J Clin Neurosci 38:8-12, 2017

5. Chowdhury FH, Haque MR, Sarkar MH, Chowdhury SM, Hossain Z, Ranjan S: Brain abscess: surgical experiences of 162 cases. Neuroimmunol Neuroinflamm 2:153-161, 2015

6. Dando SJ, Mackay-Sim A, Norton R, Currie BJ, St John JA,
Ekberg JA, et al: Pathogens penetrating the central nervous system: infection pathways and the cellular and molecular mechanisms of invasion. Clin Microbiol Rev 27:691-726, 2014

7. Dashti SR, Baharvahdat H, Spetzler RF, Sauvageau E, Chang SW, Stiefel MF, et al: Operative intracranial infection following craniotomy. Neurosurg Focus 24(6):E10, 2008

8. Duarte MJ, Kozin ED, Barshak MB, Reinshagen K, Knoll RM, Abdullah KG, et al: Otogenic brain abscesses: a systematic review. Laryngoscope Investig Otolaryngol 3:198-208, 2018

9. Eisenberg MB, Lopez R, Stanek AE: Abscess formation within a parasagittal meningioma. Case report. J Neurosurg 88:895-897, 1998

10. Erdoğan E, Cansever T: Pyogenic brain abscess. Neurosurg Focus 24(6):E2, 2008

11. Erdogan E, Izci Y, Dizer U, Baysefer A: Multiple brain abscesses in a baby: case report and literature review. Ann Neurosurg 2:1-6, 2002

12. Esplin N, Stelzer JW, All S, Kumar S, Ghaffar E, Ali S: A case of streptococcus anginosus brain abscess caused by contiguous spread from sinusitis in an immunocompetent patient. Cureus 9:e1745, 2017

13. Foerster BR, Thurnher MM, Malani PN, Petrou M, CaretsZumelzu F, Sundgren PC: Intracranial infections: clinical and imaging characteristics. Acta Radiol 48:875-893, 2007

14. French H, Schaefer N, Keijzers G, Barison D, Olson S: Intracranial subdural empyema: a 10-year case series. Ochsner J 14:188-194, 2014

15. Gadgil N, Patel AJ, Gopinath SP: Open craniotomy for brain abscess: a forgotten experience? Surg Neurol Int 4:34, 2013

16. Goto Y, Ebisu T, Mineura K: Abscess formation within a cerebellar metastasis: case report and literature review. Int J Surg Case Rep 10:59-64, 2015

17. Hakan T: Management of bacterial brain abscesses. Neurosurg Focus 24(6):E4, 2008

18. Helweg-Larsen J, Astradsson A, Richhall H, Erdal J, Laursen A, Brennum J: Pyogenic brain abscess, a 15 year survey. BMC Infect Dis 12:332, 2012

19. Jho DH, Spiliopoulos K, Stein TD, Williams Z: Concomitant presentation of a glioblastoma multiforme with superimposed abscess. World Neurosurg 75:126-131, 50-53, 2011

20. Kural C, Ozer MI, Ezgu MC, Mehtiyev R, Yasar S, Kutlay AM, et al: Intracavitary amphotericin B in the treatment of intracranial aspergillosis. J Clin Neurosci 51:75-79, 2018

21. Lai PH, Ho JT, Chen WL, Hsu SS, Wang JS, Pan HB, et al: Brain abscess and necrotic brain tumor: discrimination with proton MR spectroscopy and diffusion-weighted imaging. AJNR Am J Neuroradiol 23:1369-1377, 2002

22. Lange N, Berndt M, Jörger AK, Wagner A, Wantia N, Lummel N, et al: Clinical characteristics and course of primary brain abscess. Acta Neurochir (Wien) 160:2055-2062, 2018

23. McIntyre PB, Lavercombe PS, Kemp RJ, McCormack JG: Subdural and epidural empyema: diagnostic and therapeutic problems. Med J Aust 154:653-657, 1991

24. Moosa S, Ding D, Mastorakos P, Sheehan JP, Liu KC, Starke RM: Endoport-assisted surgical evacuation of a deep-seated cerebral abscess. J Clin Neurosci 53:269-272, 2018

25. Muzumdar D, Jhawar S, Goel A: Brain abscess: an overview. Int J Surg 9:136-144, 2011

26. Parikh V, Tucci V, Galwankar S: Infections of the nervous system. Int J Crit Illn Inj Sci 2:82-97, 2012

27. Razzaq AA, Jooma R, Ahmed S: Trans-mastoid approach to otogenic brain abscess. J Pak Med Assoc 56:132-135, 2006

28. Sarmast AH, Showkat HI, Bhat AR, Kirmani AR, Kachroo MY, Mir SF, et al: Analysis and management of brain abscess; a ten year hospital based study. Turk Neurosurg 22:682-689, 2012

29. Toh CH, Wei KC, Chang CN, Ng SH, Wong HF, Lin CP: Dif- 
ferentiation of brain abscesses from glioblastomas and metastatic brain tumors: comparisons of diagnostic performance of dynamic susceptibility contrast-enhanced perfusion MR imaging before and after mathematic contrast leakage correction. PLoS One 9:e109172, 2014

30. van Gijn J: Cerebral venous thrombosis: pathogenesis, presentation and prognosis. J R Soc Med 93:230-233, 2000

31. Watson C, Post N, Camacho A: Subdural empyema mimicking subacute subdural hematoma on CT imaging. Interdiscip Neurosurg 13:92-94, 2018

\section{Disclosures}

The authors report no conflict of interest concerning the materials or methods used in this study or the findings specified in this paper.

\section{Author Contributions}

Conception and design: Izci. Acquisition of data: all authors. Analysis and interpretation of data: Izci, Kural, Kirmızigoz, Ezgu. Drafting the article: Izci, Bedir, Kutlay. Critically revising the article: Kural, Kutlay. Reviewed submitted version of manuscript: Izci, Bedir. Study supervision: Izci, Kutlay.

\section{Correspondence}

Yusuf Izci: University of Health Sciences, Gulhane School of Medicine, Ankara, Turkey. yusufizci@yahoo.com. 